\title{
PLASTIC BRONCHITIS IN A 19-MONTH-OLD BOY WITH GLENN-STAGE CONGENITAL HEART DISEASE. CASE REPORT AND LITERATURE REVIEW.
}

LUCÍA SANJUÁN BENITA ${ }^{1}$, Sara De la Mata Navazo ${ }^{1}$, Julia Garcia Mancebo ${ }^{1}$, and Laura Butragueño Laiseca ${ }^{1}$

${ }^{1}$ General University hospital Gregorio Maranon

October 14, 2020

\begin{abstract}
Plastic bronchitis is an uncommon condition with a poor prognosis characterized by an obstructive respiratory pattern due to airway blockage by the presence of bronchial casts. Therefore, it is important to include it in the differential diagnosis of respiratory distress, particularly in patients with underlying pathologies predisposing to this condition. The etiology is multifactorial, but typically occurs in children with congenital heart disease, particularly post-Fontan procedure. The treatment is based on improving the underlying condition, which can be challenging in this type of patients.
\end{abstract}

\section{PLASTIC BRONCHITIS IN A 19-MONTH-OLD BOY WITH GLENN-STAGE CONGENI- TAL HEART DISEASE. CASE REPORT AND LITERATURE REVIEW.}

Authors:

Lucía Sanjuán Benita, MD $(1,2)$

Sara de la Mata Navazo, MD $(1,2)$

Julia García Mancebo, MD $(1,2)$

Laura Butragueño Laiseca, MD $(1,2,3,4)$

Affiliations:

1. Pediatric Intensive Care Deparment, Gregorio Marañón General University Hospital, Madrid, Spain.

2. School of Medicine Complutense University of Madrid, Madrid, Spain.

3. Gregorio Marañón Health Research Institute, Madrid, Spain,

4. Mother-Child Health and Development Network (RedSAMID) of Carlos III Health Institue, Madrid, Spain

\section{Conflict of interest statement and source of funding}

We declare there are not any conflicts of interest and there are not any sources of financial support.

Author's contact information: Lucía Sanjuán Benita. E-mail:luciasanjuanbenita@gmail.com; Gregorio Marañón General University Hospital, Pediatric Intensive Care Unit, 48 O’Donell St.

Article word count: 1034

Dear Editor: 
We read the original article High-flow nasal cannula for the treatment of life-threatening plastic bronchitis published on your journal ${ }^{1}$. This article suggests that the use of home high-flow nasal cannula (HFNC) may be a viable therapy for life-threatening plastic bronchitis. As the authors say in the article, treatment options for plastic bronchitis are limited and not always effective. Authors defend that using HFNC could generate positive intra-airway pressure that might counteract the dilatation of pulmonary lymphatics, and therefore, the bronchial cast formation. We would like to report a case of a patient requiring chronic mechanical ventilation with life-threatening upper airway obstruction due to bronchial casts.

We report the case of a 19-month-old boy admitted to the cardiology ward with a significant past medical history for levo-transposition of great arteries with double outlet right ventricle for which the patient had undergone multiple surgical interventions including a pulmonary artery banding and a bidirectional superior vena cava to pulmonary artery shunt (Glenn procedure) at seven months old. Failure of the Glenn circulation with reverse blood flow through the shunt was observed with low cardiac output and development of venous collateral circulation (Figure 1A). Other postoperative complications included an atrioventricular blockage requiring pacemaker, chronic mechanical ventilation through tracheostomy, and massive chylothorax in the immediate postoperative period, followed by prolonged hospital course due to medical complexity from failed Glenn circulation.

Twelve months following the Glenn procedure, he suffered an episode of respiratory distress, with decreased oxygen saturation and coughing fits, coinciding with generalized swelling and one kilogram weight gain in the last days. Two of the fits ended up with spontaneous expectoration of bronchial casts (figure $1 B$ ).

He was transferred to the Pediatric Intensive Care Unit (PICU) where nebulized treatment with bronchodilators, tissue-type recombinant plasminogen activator (rTPA) and oral azithromycin was initiated as treatment for plastic bronchitis. The diuretic treatment was also optimized.

The patient experienced an initial improvement but 12 hours later he developed an episode of severe respiratory distress, with persistent desaturation to $50-60 \% \mathrm{SpO} 2$ despite manual ventilation. A chest radiography showed complete left lung collapse with right lung infiltrates. An urgent flexible bronchoscopy (FB) was performed, removing several rubbery white casts by suction and bronchoalveolar lavage with a mixture of rTPA and saline solution with improvement and increase of the oxygen saturation.

In the following days, treatment with nebulized rTPA and later with nebulized heparin was initiated. Pulmonary circulation was optimized with pulmonary vasodilators including inhaled nitric oxide and inhaled prostaglandin added to his previous treatment with bosentan and sildenafil. Follow-up FB were performed, without finding more bronchial casts.

Three cardiac catheterization procedures were performed during the following weeks in order to optimize hemodynamics: obstruction of the superior venous circulation was detected, without identifying any thrombosis in the Glenn circuit; the venous collateral circulation was obliterated; a stent was placed in the atrioventricular septum; and two stents were placed at the pulmonary banding in order to reduce the pressure gradient through the Glenn.

The patient was discharged two months later under continued heart failure management, bronchodilators, pulmonary vasodilators and nebulized hypertonic saline. During the following months, the patient presented occasionally with spontaneous bronchial cast expectoration but without respiratory distress or new plastic casts finding in repeated FB. However, he recently required a new admission at the PICU due to a new episode of respiratory distress requiring additional FB and cardiac catheterization procedure in order to optimize hemodynamics.

Plastic bronchitis is an uncommon condition consisting of obstruction of the airway by bronchial thick, rubbery, white casts. It is diagnosed after its expectoration or direct visualization during a $\mathrm{FB}^{2}$ Patients present with respiratory symptoms such as cough, dyspnea and respiratory distress. Although there are some specific tests such as the dynamic contrast magnetic resonance lymphangiography for the visualization of lymphatic abnormalities, most radiologic images are non-specific for this condition being common the 
presence of signs of bronchi obstruction.

The etiology is multifactorial with the influence of inflammation and an excessive permeability of the alveolocapillary membrane, mainly due to venous and lymphatic stasis that occurs after critical congenital heart disease surgeries. It is a rare complication in patients after Glenn shunt, more commonly reported after Fontan's surgery ${ }^{3}$ with a prevalence of $14-23 \%$ and a mortality rate up to $38 \%$. Viral infections and inflammatory pulmonary diseases like asthma are also uncommon causes of plastic bronchitis ${ }^{3}$. In our case, the patient presented with fluid overload in the days prior to the acute episode that could have increased the lymphatic pulmonary stasis in a patient with an underlying complex congenital cardiopathy, predisposing the casts formation.

Treatment of the acute condition consists of FB bronchoalveolar lavage and casts suction, with the addition in some cases of the administration of a fibrinolytic agent via FB. Other treatment options that have been described to improve the symptoms include the use of nebulized heparin and rTPA. There is not enough clinical evidence to support any of these treatments, but reports suggest that heparin has more antiinflammatory effects, less vascular bleeding risk $^{2}$, and significantly reduced treatment costs ${ }^{5}$, which were the reasons that motivated the change from rTPA to heparin in our case.

In recent years, another treatment based on the pathophysiology of the disease has gained relevance: the selective embolization of the thoracic duct that would interrupt the extravasation of the lymph and, therefore, the formation of bronchial casts. Although previous results are encouraging ${ }^{6}$, this therapy was not attempted in our patient as lymphatic canalization was previously unsuccessful to control a high output chylothorax.

Nevertheless, definitive treatment is based on improving the underlying condition, which can be challenging in this type of patients. In patients with congenital heart disease, a good cardiac output must be achieved, optimizing the hemodynamic condition by using pulmonary vasodilators, administering drugs to reduce the cardiac preload, or by performing surgical/interventional procedures as previously discussed.

In conclusion, plastic bronchitis is an uncommon disease but with poor prognosis as it represents a threat of airway obstruction and asphyxia. Therefore, it is important to include it in the differential diagnosis of respiratory distress, particularly in patients with underlying pathologies predisposing to this condition. Early treatment includes bronchial lavage through FB but definitive treatment is based on improving the underlying condition, a challenge in these patients.

Figure legend

Figure 1. A: Venous collateral circulation demonstrated by 3D tomography reconstruction. B: Bronchial cast expectorated by the patient.

\section{References.}

1. Vezina K, Dipchand AI, Narang I. High-flow nasal cannula for the treatment of life-threatening plastic bronchitis. Pediatr Pulmonol. 2020 Apr;55(4):E1-E2.

2. Rubin B.K. Plastic Bronchitis. Clin Chest Med. 2016; 37:405-408.

3. Madsen P, Shah SA and Rubin BK, Plastic bronchitis: new insights and a classification scheme. Paediatr respir rev. 2005; 6: 292-300

4. Caruthers RL, Kempa M, Loo A, et al. Demographic characteristics and estimated prevalence of Fontan-associated plastic bronchitis. Pediatr Cardiol. 2013; 34:256-61.

5. Eason DE, Cox K, Moskowitz WB. Aerosolised heparin in the treatment of Fontan-related plastic bronchitis. Cardiol Young. 2014 Feb;24(1):140-2

6. Maleux, G, Storme, E, Cools, B, et al. Percutaneous embolization of lymphatic fistulae as treatment for protein-losing enteropathy and plastic bronchitis in patients with failing Fontan circulation. Catheter Cardiovasc Interv. 2019; 94: 996-1002. 
figures/figure-1/figure-1-eps-converted-to.pdf 